\title{
ENERGÍA DE FUENTES RENOVABLES Y PROTECCIÓN DEL PAISAJE Propuesta de un modelo para la construcción de estrategias de participación de la población. Aplicación en el territorio de Ulassai y Perdasdefogu (Cerdeña)
}

\author{
Autor: Serena Lisai \\ Universidad: Università degli studi di Sassari \\ Director de la tesis en curso: Marco Dettori y Ivan Blečić \\ EMail: serenalisai@gmail.com
}

\section{RESUMEN}

En una realidad en la que, debido a lo cambio climático, la atención sobre las fuentes renovables es cada vez mayor, a nivel nacional e internacional. Estas instalaciones resultan ser una oportunidad de desarrollo tanto económica como social. En esta tesis se indaga sobre el modo en el que las Fuentes de Energía Renovable se relacionan con el paisaje, el ambiente y el desarrollo económico, social y cultural del territorio. A partir del análisis de los conflictos generados y de los instrumentos de evaluación ambiental, se identifica en la falta de implicación de la población, la causa de la fácil manipulación del debate público sobre este tema. El estudio de un contexto específico sardo permite identificar un modelo de la construcción de estrategias adecuadas para portar un conocimiento mas crítico a la población, con el objetivo de convertir a los habitantes en protagonistas del desarrollo sostenible de su proprio territorio.

Palabras clave: Fuentes renovables, paisaje, desarrollo sostenible, participación

\begin{abstract}
Nowadays, due to the climate change, the importance of use renewable sources is increasing, both at small and large scale. Solar, wind or biomass project and design seems to be an opportunity to develop economically and socially. This thesis investigates the ways in which the Renewable Energy Production can be related to the landscape, the environment and the economic, social and cultural development of a territory. An analysis of the generated conflict and environmental assessment tools shows the lack of involvement of population as the reason of the easy manipulation of the public debate on the topic of renewable energies. The study of a specific Sardinian context will help to identify a model to build the most appropriate strategies to build a critical knowledge in the population that makes people protagonist of the sustainable development of its own territory.
\end{abstract}

Key words: Renewables, landscape, sustainable development, participation 


\section{INTRODUCCIÓN}

Durante los últimos decenios el planeta Tierra ha sido maltratado a través de una serie de calamidades que la ciencia ha, lamentablemente, atribuido a los cambios climáticos. Cuando se habla de cambio climático se refiere a un cambio del valor medio o de la inestabilidad de las propiedades del clima, que persiste durante un mismo periodo, desde hace décadas o intervalos todavía más largos (Cubash et al. 2013). El sistema climático de la tierra se basa en el equilibrio, que se preserva gracias a una serie de intercambios de energía entre la superficie terrestre y la atmósfera: la energía que llega del sol debe ser ecuánime a las radiaciones que van en sentido contrario. Si la Tierra absorbe del sol más energía de la que libera, la temperatura aumenta: la llegada de un balance energético consiente la vida. La ruptura de este equilibrio ha causado la sucesión de periodos de glaciaciones y periodos caracterizados por altas temperaturas. Los científicos coinciden en que después de la Revolución Industrial del siglo XVIII las causas del cambio climático no pueden ser atribuidos solamente a la naturaleza sino que el elemento antrópico ha adquirido el rol protagonista (IPCC, 2013). Una de las principales consecuencias que la actividad humana tiene es el incremento de las concentraciones de los llamados gases invernadero en la atmósfera. El nivel de estos gases, ya presentes en la naturaleza, ha incrementado notablemente por actividades humanas como la producción industrial, el sistema de transporte o la producción agrícola.

Además, existe una fuerte conexión entre la actividad antrópica y el cambio de la reflexión de la superficie terrestre: en el último siglo la capacidad de la tierra de reflejar los rayos solares ha claramente disminuido causando una mayor absorción de éstos y, como consecuencia, un aumento considerable de la temperatura. La mayor parte de los componentes de la comunidad científica concuerda a nivel mundial sobre la manera en la que el modelo de crecimiento basado en la explotación de las fuentes fósiles, está produciendo un efecto devastante en varias regiones del mundo. Pasqualetti (2012) identifica en la variación del funcionamiento del sistema económico el punto inicial para una transformación total del modelo de desarrollo social sucesivos en el tiempo. Actualmente los científicos están de acuerdo sobre la necesidad de una importante reforma del modelo de desarrollo económico de manera que sea patente una verdadera y propia transición energética hacia una nueva fase basada en las fuentes renovables (FER). A mediados de los años 90' el economista Jeremy Rifkin (2011: 10) definió el concepto de Tercera Revolución Industrial para indicar la necesidad de ir en "busca de un nuevo paradigma económico que pueda ser acompañado hacia una era post-carbón". El objetivo es utilizar las nuevas tecnologías para construir un progreso energético sostenible basado en la dimisión de las fuentes tradicionales fósiles y en la mejora de las energías renovables.

\section{OBJETIVO DE LA INVESTIGACIÓN}

Este trabajo de tesis propone como objetivo la construcción de las estrategias que afronten los conflictos generales en el territorio, a partir del desarrollo de las tecnologías FER, entre paisaje, ambiente y desarrollo de la sociedad humana; identificando de manera realista la implicación de la población como elemento clave para garantizar un desarrollo sostenible en el contexto local.

El presente trabajo de tesis se pone como objetivo la construcción de una serie de estrategias territoriales que pongan el proyecto de nuevos distritos energéticos renovables en relación con el paisaje y con el contexto social, económico y cultural en el territorio sardo. Para realizar esto se han estudiado las relaciones existentes entre las nuevas tecnologías de energía alternativa, el paisaje, el ambiente y el desarrollo de la sociedad. Se utilizará un enfoque que integra varios aspectos del territorio y activa procesos de participación de la población, no sólo en las fases de proyección, sino anteriormente a los procesos de evaluación ambiental, construyendo en la comunidad el conocimiento y la sensibilidad crítica sobre el tema de las fuentes renovables y de la tutela y desarrollo del propio territorio.

\section{LOS TEMAS DE LA INDAGINE}

La relación entre las nuevas tecnologías de producción de energía a través de fuentes renovables y los diversos elementos que constituyen el paisaje han hecho emerger una serie de conflictos que ponen a la planificación delante de un nuevo reto. La realidad sarda ha sido a menudo escenario de decisiones 
equivocadas de la planificación, que han provocado situaciones de fuerte contraste entre los diferentes elementos del territorio. En los últimos años, la entrada en el campo de las fuentes de energía alternativas ha causado numerosos debates animando la cuestión de qué es lo que habría que defender, si el paisaje, el trabajo, la salud o el ambiente. Para entender de que manera estos elementos entran en relación, ha sido necesario especificar el sentido y la definición de los que vienen tratados en este trabajo.

\subsection{Paisaje}

El cambio en la concesión de paisaje ha sido flanqueada por una evolución en los carácter de la normativa nacional y europea. Con la Convención Europea del Paisaje de Florencia en el 2000 y con el artículo 131 D. Igs 22 Enero 2004, n.42 se afirmó el carácter colectivo del paisaje, definido como "una parte homogénea de territorio cuyos caracteres derivan de la naturaleza, de la historia humana o de las recíprocas interacciones" (D.Igs n. 42 del 2004, art. 131, subpárrafo 1) y legando la tutela y los "aspectos y caracteres que constituyen la representación material y visible de la identidad nacional, en cuanto a la expresión del valor cultural" (comma 2). Por lo tanto queda aclarada la estrecha conexión entre paisaje y comunidad, no solo el que lo habita, sino por todo aquel que se beneficia de cualquier manera y por cualquier periodo de tiempo. La identidad de los lugares entra en juego atribuyendo al paisaje un carácter casi espiritual (Ferraro, 2012). La Convención introduce una serie de innovaciones sobre las políticas de gestión del paisaje, extendiendo las acciones no solo a la salvaguardia del paisaje existente si no también a la planificación a través de "acciones fuertemente previsoras para la mejora, rehabilitación y a la creación de paisaje" (art. 1).

Además han entrado en el campo de la aplicación "los paisajes de la vida cotidiana y los paisajes degradados" (art. 2) superando totalmente el carácter subjetivo de belleza utilizado tradicionalmente para indicar un lugar digno de tutela. Ya Sereni (1984), superando el simple carácter estético describe el paisaje como la sedimentación de una serie de relaciones culturales, de propiedad y de producción que permanecen en el tiempo constituyendo el punto de partida para sucesivas transformaciones.

El modo de relacionarse con el paisaje se constituye ya sea de elementos que unifican los objetos que forman parte del patrimonio biológico innato (Kaplan, 1979) como de elementos totalmente subjetivos debido a las experiencias y a los recorridos formativos de algún individuo. Los beneficios que una persona obtiene están fuertemente conectados con la percepción subjetiva que tiene (Bourassa, 1990).

\subsection{Ambiente}

Así como se describe en el Diccionario de Ecología (Di Fidio, 1966), el ambiente es "el conjunto con factores abióticos (físicos y químicos) en los cuales viven los diversos organismos y en particular el hombre. Pero haciendo referencia específica a la sociedad humana el ambiente ha alcanzado un significado más amplio: es todo lo que respecta al hombre, lo puede influenciar y, viceversa, puede ser influenciado. El valor que hoy en día le viene atribuido resale a los últimos decenios, hasta tal punto ha sido solo indirectamente contemplado al interno de la Constitución del 1948 en el artículo 9 y 32. Se hace referencia de hecho a dos tipos de necesidad: la primera conectada a la protección de la "naturaleza por lo que es", la segunda ligada al concepto de ambiente salubre, necesario para favorecer una mejor cualidad de la vida de las personas. Como consecuencia de la protección del ambiente no posee ninguna finalidad estética sino que se refiere a la exigencia de mantener un hábitat que garantice un elevado nivel de cualidad de la vida de las personas.

La misma Corte Constitucional ha llegado a definir "la salvaguardia del ambiente como derecho fundamental de la persona e interés de la comunidad" (sent. n. 641/1987, punto 2.2) considerando de esta manera el paisaje como elemento constituyente del ambiente. Se subraya el carácter pluridimensional, haciéndoles entrar dentro de la misma definición ya sea los aspectos ligados a las condiciones ambientales como a la calidad de la vida, no solo desde un punto de vista sanitario sino además desde un punto de vista cultural, educativo y de participación (Ferraro, 2012). 


\subsection{Análisis de conflictos}

En un escenario en el cual los cambios climáticos están poniendo a la especie humana frente a continuos retos por la supervivencia, parece fundamental la carrera por las medidas, no sólo de adaptación, sino de verdadera y propia prevención contra los efectos más devastadores e irreversibles. La modificación del sistema de producción de energía es sólo una entre las medidas adoptadas pero abre camino a las acciones preventivas de graduales transformaciones en el comportamiento de las personas. En el campo de la planificación la necesidad de aspirar a una transición energética se traduce en un ulterior reto para llevar a cabo políticas y acciones que comporten una mejora de la cualidad del ambiente global sin destruir las características del territorio a nivel local. La inserción de las nuevas tecnologías de producción de energía de fuente renovables es una tema de estudio que ofrece innumerables ideas, más o menos exploradas en el campo de la planificación (Möller, 2003; Sukkumnoed, 2003; Peterson, 2006), de la arquitectura del paisaje (Koh, 2013; Stremke y Koh, 2011) y de la ingeniería (Yüksel, 2007).

En este trabajo de tesis se ha decidido mantener en un segundo plano los impactos en el paisaje de las tecnologías renovables, concentrando la atención en el efecto que estas instalaciones generan en la organización de la sociedad y en las personas, identificando es estas últimas el elemento principal del cual es necesario partir para llevar a cabo una verdadera y propia transición energética. Mirando la modificación del sistema de producción de energía como un camino gradual, pero necesario, la investigación relativa al impacto sobre las personas constituye un primer paso para la construcción de políticas de gestión del cambio climático.

\subsubsection{Paisaje y ambiente}

Aunque, como se ha dicho anteriormente, en la lectura de ambiente y paisaje resultan formar parte de un mismo sistema, cuando el campo de la investigación es la instalación en el territorio de sistemas de energía renovable, es fácil asistir a un colisión entre los dos elementos (Ferraro, 2012). En el ámbito de la Evaluación de Impacto Ambiental, uno de los elementos mayoritarios que se opone a la aprobación de un proyecto FER es la incompatibilidad paisajística del mismo proyecto. El conflicto es evidente si se mira a este tipo de proyecto por una parte, como medio de tutela del ambiente porque se está produciendo "energía limpia", y por otra como choque directo de esta idea por la necesidad de eligir un lugar donde la productividad sea máxima. Está claro, por ejemplo, que la construcción de una instalación eólica tiene como finalidad la disminución de las emisiones contaminantes, así como alcanzar los objetivos europeos de lucha contra el cambio climático; pero por otro lado las posiciones geográficas dotadas de mayor viento, por lo tanto idóneas, son las cordilleras, las colinas y las montañas, en las cuales la inserción de una instalación de este tipo provocaría un impacto visual, motivo principal del cambio del paisaje.

\subsubsection{Paisaje y desarrollo}

Como tradicionalmente el desarrollo industrial ha puesto a la sociedad de frente a la necesidad de elegir si atribuir mayor importancia a la creación de puestos de trabajo, y por lo tanto, a un desarrollo económico y social, o a la tutela del paisaje y de un ambiente salubre, hoy el proceso y las fuentes renovables ponen nuevamente estos elementos en conflicto entre ellos. Como sujeto central al interno de esta tipología de conflicto resulta ser la población que, sobre la base de la propia consciencia y actitud, propende por la seguridad de un cierto elemento más que por el otro. En el panorama sardo se ha asistido a casos en los que los mismos ciudadanos si han unido oponiéndose a la aprobación o a la construcción de instalaciones FER reconociendo en éstas un impacto negativo para el paisaje que constituye su propia identidad. Son ejemplo las experiencias como las de la comunidad sarda de Cossoine y Buddusò que, apelándose a los resultados de un referéndum consultivo, han formado comités populares en oposición a la construcción de nuevas instalaciones de producción de energía, respectivamente termodinámica y biomasa. Contemporáneamente, a causa de la crisis económica y a las condiciones precarias en las cuales muchas realidades sardas les toca vivir, se ha asistido a la actitud opuesta: la posibilidad de construcción de una nueva instalación ha hecho nacer, sobretodo entre los jóvenes, una fuerte esperanza y como consecuencia su total apoyo. El posible impacto paisajístico se coloca en un segundo plano respecto a la necesidad de activar en la comunidad una serie de procesos de desarrollo económico y social. 


\subsubsection{Ambiente y desarrollo}

Durante el periodo de constante crecimiento económico precedente a la Segunda Guerra Mundial, se ha reconocido la idea de que sólo garantizando primero determinados ritmos de desarrollo económico y manteniendo altos niveles de inversión, hubiera sido posible perseguir el objetivo de mejorar las cualidad del ambiente. Volviendo a una reflexión de Salzano (1998) que resulta ser todavía actual, el significado positivo atribuido al término desarrollo tiene necesidad de ser separado del término crecimiento. Generalmente, al contrario, el desarrollo pide que la crecida de algunos elementos de la tradición económica se detengan. La misma Comunidad económica europea ha afirmado que a largo plazo la protección de los recursos ambientales será una condición de base para el sano crecimiento económico, la cual a su vez contribuirá para el mejorar el ambiente (Salzano, 1998). La conocida relación entre la competencia internacional y la cualidad de la vida y del ambiente marca este tema esencial dentro de las decisiones de planificación. En algunos casos la necesidad de llevar a cabo una una transición energética hacia las fuentes renovables no es una sentimiento difuso en las comunidades, las cuales han impreso su propio desarrollo económico en campos como el turismo o la agricultura. La inserción de estas nuevas tecnologías puede generar conflictos con actividades antiguamente radicadas en el contexto, $y$, como consecuencia, con aquellos que se sustentan gracias a esas actividades.

\subsection{La planificación en la gestión de conflictos}

El urbanista tiene que gestionar estos conflictos de manera que la real necesidad de llevar a cabo una transición energética (Magoni, 2013) hacia las fuentes de energía alternativa, non se traduzca en un empobrecimiento del paisaje y que una aproximación excesivamente vinculante no impida el desarrollo de territorios donde las FER constituyen una alternativa eficaz respecto a modelos de desarrollo poco adecuados. En el campo energético, el elemento principal de la política pública, se convierte en el principio de integración (Ferraro 2012) entre el interés ambiental y paisajístico, garantizando el desarrollo del territorio. La gestión de los conflictos es posible a través de una nueva idea de proyecto de paisaje, en el cual la instalación de producción energética pasa a ser un elemento del paisaje mismo, contribuyendo al reconocimiento de la especificidad mediante una relación coherente con el contexto. La balanza entre paisaje, ambiente y desarrollo y, por lo tanto, la superación de los conflictos es el elemento clave para alcanzar un desarrollo sostenible. Se hace en este caso referencia a la definición dada por la ONU en un informe de la Comisión Mundial para el Medioambiente y el Desarrollo, donde se entiende la satisfacción de las "necesidades de la actual generación sin comprometer la capacidad de aquellas futuras de responder a las suyas" (AA.VV. 1989: 32), pero pretendiendo en préstamo el propuesta de Salzano (1998) que habla más que de un mantenimiento de las condiciones actuales, de su mejoramiento. Al mismo tiempo es deber de los urbanistas y paisajistas aquel de propender por el desarrollo sostenible del propio territorio garantizando el respeto de los caracteres propios del paisaje.

\subsubsection{El instrumento de Evaluación del Impacto Ambiental}

El instrumento que actualmente, ya sea a nivel nacional que regional, intenta encontrar una solución a estos conflictos es la Evaluación del Impacto Ambiental (VIA, en Italia). Del análisis de sus propios principios esta claro el tentativo de proponer las bases para la superación de los conflictos (D. Lgs. 152/2006):

- Principio de la acción ambiental: "la tutela del ambiente y de los ecosistemas naturales y del patrimonio cultural debe ser garantizada por todas las entidades públicas y privadas y de las personas físicas y jurídicas, públicas o privadas mediante acciones de precaución, prevención y corrección, y el segundo principio <<quien contamina paga>>" (art. 3-ter).

- Principio de desarrollo sostenible: "cada actividad humana debe conformarse a este principio para garantizar la satisfacción de las necesidades de las generaciones actuales sin comprometer aquellas de las generaciones futuras" (art. 3-quater).

- Principio de la subsidiariedad y leal colaboración: "pone en relación los actores que se ocupan del gobierno del territorio en todos los niveles, del Estado a las Regiones o a las pequeñas entidades locales" (art. 3quinquies). 
La VIA es un instrumento que intenta introducir a la proyección un nuevo acercamiento que pueda influenciar el proceso de decisiones, conduciéndolo además de panera participativa con la población interesada. Describe, identifica y evalúa los efectos directos e indirectos de un proyecto sobre la salud humana y alguno de sus componentes ambientales como fauna, flora, suelo, aguas, aire, clima, paisaje, patrimonio cultural y su recíproca relación.

\subsubsection{El rol de la planificación paisajística}

La relación entre los temas de la energía y del paisaje representan la base para la construcción de nuevos modelos de desarrollo "capaces de reinterpretar las razones profundas del territorio, los principios ambientales, sociales y culturales, como puntos de apoyo para una reescritura crítica de lo existente de manera que se piense en escenarios a largo plazo donde la cualidad específica de los lugares readquiera un rol estratégico" (Marchiagiani a Prestamburgo, 2010: 11). Un paso fundamental es la definición del acercamiento con el cual las políticas y las decisiones de planificación ha afrontado el tema de la energía renovable. Magoni (2013) define cuatro tipologías de criterio con las que es posible afrontar el proyecto en la óptica de una gestión con la visión de conflicto entre paisaje e innovación energética.

- Enfoque conservativo: los paisajes son clasificados en buenos y degradados, individuando una serie de soluciones de tipo vinculante que prevén la conservación de los primeros y más fácil transformación de aquellas que, lamentablemente, están comprometidos.

- Enfoque técnico: la necesidad de producir energía, aunque sea limpia, se pone sobre cualquier otro elemento del territorio, legitimando todo género de intervención, sobretodo en áreas con escaso valor paisajístico.

- Enfoque integrado: la relación entre energía y paisaje se reinterpreta, sosteniendo proyectos en los cuales los aspectos visuales, funcionales, productivos, espaciales y de gestión de las nuevas tecnologías son integrados son los elementos del paisaje, ya sean físicos o inmateriales, conectando esto al aspecto identitario y cultural del lugar.

- Enfoque refundado: se sostienen soluciones no sólo tipológicas, figurativas, espaciales y materiales sino también sociales, favoreciendo procesos de sensibilización en los temas energéticos y ambientales, de modo que se active la construcción de comportamiento virtuosos.

Un deber de la planificación es aquel de guiar la transición energética no exclusivamente a través de medidas de mitigación y adaptación sino activamente instrumento de prevención basados en un verdadera implicación de la población en esta fase de cambios.

\subsubsection{La implicación de la población}

El objetivo de la modificación del modelo de desarrollo energético non puede prescindir de un cambio en las costumbres y comportamientos de cada individuo. Bajo este punto de vista la población, y por lo tanto la atención que la planificación debe poner en ella, asume un carácter principal. Ya en el 1979 el profesor Jon Van Til (1979) anunciaba la llegada de una crisis energética que habría puesto la disciplina del gobierno del territorio de frente a la necesidad de gestionar la más grande transformación de la organización de la sociedad de la historia. Según Van Til, los instrumentos imprescindibles para gobernar este cambio son "early warmimg, brillant planning, great leadership and a rare sense of commitment and compassion among all citizans" (Van Til, 1979, p.328). La referencia es a las acciones activas de cada individuo en particular para lograr llevar a cabo los impactos de la sociedad siempre más sostenibles. Con este objetivo se llama a la planificación a inserir la implicación de la población al centro de los propios objetivos y de las propias acciones.

En referencia a la fase de consulta prevista para cualquier proyecto de construcción (o ampliación) de una instalación de energía renovable, este proceso de implicación o de participación parece difícil e incluso contraproducente. Si hipnotizamos el encuentro con el público para la presentación de un nuevo proyecto de parque eólico en un "Ayuntamiento X" de Cerdeña, nos encontramos de frente a una serie de posibles situaciones, todas unidas por una notable intensidad emocional. Cada individuo, incluyo aquellos más cercanos a la concepción del homo economicus, basa las propias acciones y las propias opiniones sobre 
elementos ligados a la irracionalidad: emociones, cuestiones éticas y necesidades sociales (Sijmons y Van Dorst, 2012). En este sentido los defensores de la racionalidad identifican los límites en la implicación de la población en los procesos de decisiones porque, debido a la fácil manipulación de las opiniones personales, en muchas ocasiones se priva de un real conocimiento sobre el tema. Para facilitar la comprensión y el objetivo final definido por este trabajo de tesis, los posibles escenarios con sus innumerables matices en base al nivel de participación, pueden ser reagrupados en dos tipologías.

En un primer caso los técnicos seleccionados por el Proponedor explicarán las principales características del proyecto delante a una platea escéptica y prevenida respecto a una nueva tecnología que no hará otra cosa que degradar y modificar el paisaje circundante. La Sociedad Proponedora es vista como fuera de toda moralidad, depredadora del paisaje, ávida y con una peligrosa confianza en el progreso (Smits, 2002). Los técnicos describen el propio proyecto como solución a innumerables problemas energéticos y económicos del territorio pero, por otra parte, la población atribuye a esa misma tecnología la generación de impactos adversos e imprevisibles.

En el segundo caso una sala llena de personas asistirá en silencio a la presentación del proyecto por parte de la Sociedad Proponedora, realizando observaciones sobre la necesidad de construir la instalación en ese territorio, porque tienen más necesidad de los anteriores de un empuje económico a nivel local (Mura y Strazzera, 2013). El rol principal en la presentación será asignado a los beneficios en términos ocupacionales de la aprobación del proyecto que portará en el territorio, despertando el interés de todas la caras jóvenes de la población.

Parece evidente que la fase de consulta, así como la propone la normativa, está dotada de límites debido a la fácil posibilidad de manipulación de la percepción y de las consecuentes disposiciones de la población. Ésto puede ser asociado al hecho que el proceso de participación sea inmerso en la fase de proyecto bajo una forma de simple presentación, durante la cual todos los "dónde", "cómo", y "por qué" emergen simultáneamente provocando un sentido de confusión (Sijmons y Van Dorst, 2012). Resulta necesario transformar la simple petición pública en un proceso participativo, activo sobre el campo, introduciendo a la persona en la temática de las energías renovables a través de la experiencia, en una fase precedente a la presentación del proyecto. Un deber de la planificación es este nuevo campo del paisaje tecnológico es aquel de interpretar las diferentes voces de la población, gobernando los sentimientos instintivos pero sin ignorarlos. La real participación pública en los procesos actuales ofrece perspectivas de superación de los miedos y de las dudas, reduciendo la posibilidad de manipulación y mejorando la capacidad de la población de evaluar de manera crítica un proyecto de modificación del propio territorio.

\section{METODOLOGÍA}

El estudio de los fenómenos de posibles manipulaciones durante la fase de presentación al público de los proyectos de instalaciones FER, es imprescindible del conocimiento específico del contexto, para poder identificar las particulares estrategias de gestión de los conflictos, diferentes para cada ámbito. Se ha tenido la posibilidad de conocer la realidad de los ayuntamientos de Ulassai y Perdasdefogu durante la participación con el Servizio Valutazione Ambientale a la presentación al público de un proyecto de ampliación del parque eólico. El campo de la investigación resulta ser interesante para el análisis del comportamiento de la población de los pueblos anteriormente nombrados.

Se ha decidido adoptar el instrumento del cuestionario, el cual ha sido suministrado personalmente, y no a través de internet, para tener la posibilidad de entrar en contacto con el contexto y alcanzar, de manera casual, los diferentes sectores de la población. Las preguntas intentan definir cada individuo sobre la base de tres factores principales: el conocimiento, la posición y la disponibilidad a la participación.

La primera parte ha sido una investigación a nivel de conocimiento de los entrevistados sobre las tecnologías renovables, diferenciando entre percibida, a través de preguntando de auto-evaluación y la real, a través de un "examen" (verdadero/falso).

La segunda parte define la posición (a favor/en contra) de la población respecto a las fuentes renovables, indagando sobre las motivaciones y las percepciones de las instalaciones y de los beneficios. Para entender el nivel de importancia que se atribuye al tema de la salvaguardia del paisaje y del medioambiente, del 
desarrollo económico y la producción de puestos de trabajo, los entrevistados han sido llamados a evaluar tres casos hipotéticos de construcción de instalaciones eólicas, cada uno con diferente impacto y relativos beneficios.

La última parte del cuestionario sirve para definir el nivel de disponibilidad para escuchar y ampliar el propio conocimiento para poder identificar las mejores acciones de implicación de la población.

La investigación cognitiva así estructurada tiene como objetivo indagar sobre el real conocimiento de la población en el tema de producción de energía renovable, analizando los elementos que pueden entrar en conflicto con la base de las instalaciones y de los beneficios percibidos. Una vez definidos los procesos que la construcción del parque eólico ha generado en los diferentes contextos, será posible indicar de manera más específica la modalidad de implicación de la población. Se busca una relación entre las actitudes de la población y el contexto habitado, el cual en ambos casos resulta ser influenciado, positivamente o negativamente, de la construcción de la instalación FER.

\section{RESULTADOS Y CONSIDERACIONES}

La fase de investigación en el campo ha sido fundamental para delinear este trabajo de tesis, la inmersión personal en el contexto ha permitido entender e interpretar los procesos sociales en curso, invisibles a mera observación a través de medios cartográficos. La experiencia en Ulassai y Perdasdefogu ha permitido la recogida de información de elevada cualidad para la búsqueda, garantizando una fotografía más precisa de la realidad hallada respecto a una búsqueda online.

\subsection{El descubrimiento del contexto}

El territorio analizado se sitúa en el interior de la región histórico-geográfica de Ogliastra, en la provincia de Nuoro, en la parte centro-orienta de Cerdeña. Previamente al trabajo en el campo, el territorio ha sido analizado a través de la cartografía, la cual ha permitido de clarificar la localización de los dos ayuntamientos protagonistas de la investigación, Perdasdefogu y Ulassai. La conformación física del territorio es fundamentalmente para comprender los motivos de la base de la diferente distribución de las instalaciones y de los beneficios relativos a la presencia de un parque eólico (Gráfico 1).

Los dos ayuntamientos de Ulassai y Perdasdefogu cuentan con una población de, respectivamente, 1.465 y 1.957 habitantes. Aunque estén ambos dotados de un número de habitantes bastante reducido, el territorio del interior de los límites administrativos cuenta con un total de más de 20.000 ha. (respectivamente 12.331 ha y $7.708 \mathrm{ha})$.

La construcción del "Parque eólico de Ulassai" en el 2005 ha puesto indirectamente a estas dos realidades en relación. La instalación de producción de energía eólica está compuesto por 48 aerogeneradores, con una potencia total instalado de $96 \mathrm{MW}$. Como se puede observar en el gráfico 1, la localización geográfica insiste en el territorio entre el pueblo de Ulassai y Perdasdefogu, pero administrativamente permanece en el interior de los límites ulasseses. La conformación geomórfica es un elemento fundamental en la construcción de las dinámicas sociales consecuentes a la apertura de la instalación: la vista de las palas es posible solo desde el centro urbano de Perdasdefogu, mientras la montaña de Ulassai protege el centro habitado del impacto visual que genera el parque (Gráfico 2). Durante estos años, por lo tanto, la generación de empleo (alrededor de 30 personas) ha interesado exclusivamente al ayuntamiento de Ulassai. Además, la localización del parque en un área de uso cívico ha generado importantes beneficios económicos para la administración ulassese, que pueden llegar al millón de euros al año. La construcción del parque ha permitido al Ayuntamiento la financiación de una serie de iniciativas culturales y sociales. Por otra parte, precedentemente a la construcción del parque, a través de una recogida de firmas, los habitantes de Perdasdefogu se han opuesto a la ampliación del proyecto del parque en su territorio, por miedo al impacto paisajístico que habrían generado. Hoy en día este hecho se recuerda con remordimiento a causa de los beneficios económicos perdidos que, al contrario, habrían permitido al pueblo de tener una importante fuente de sustento. 


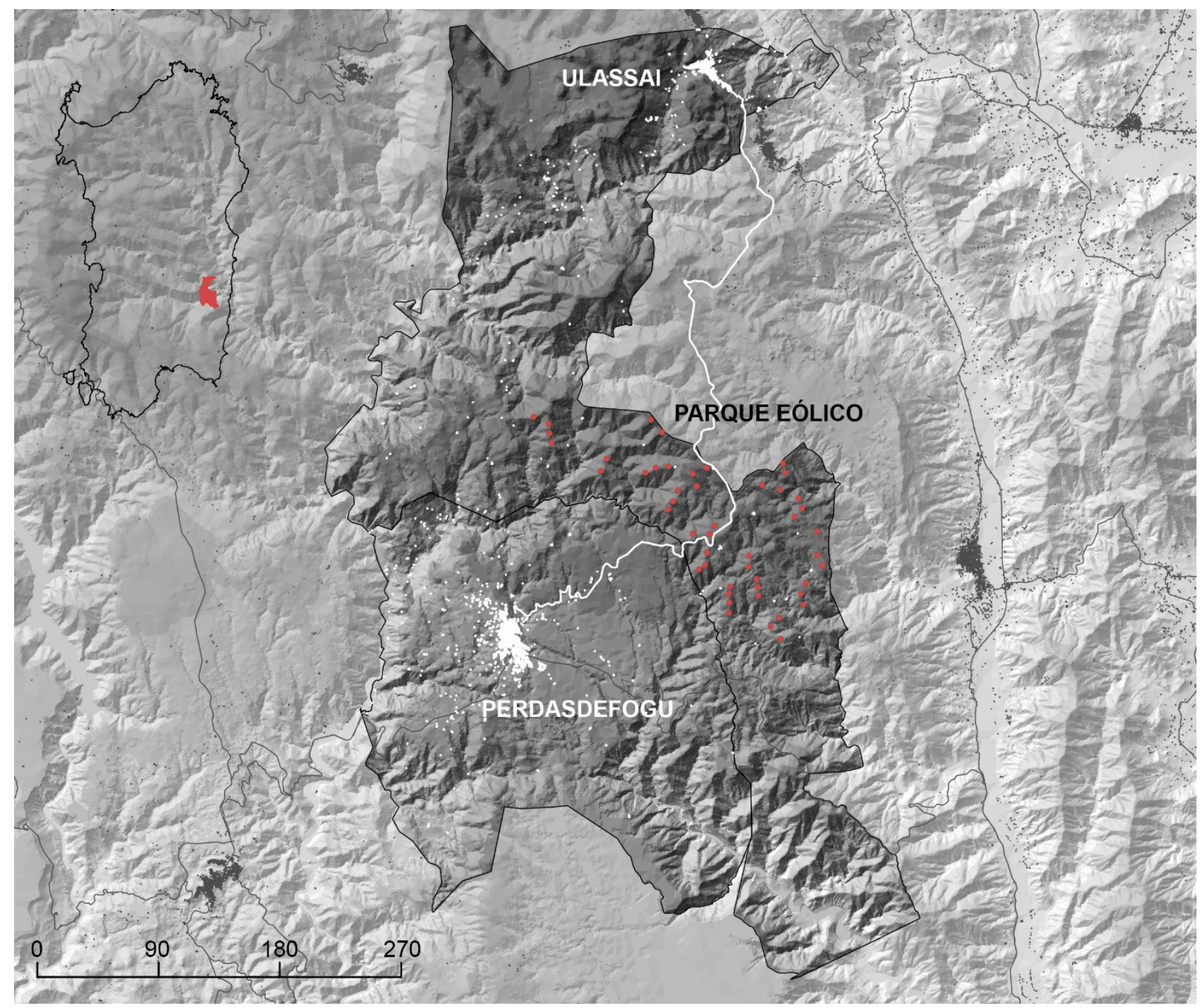

Gráfico 1: Los pueblo de Ulassai y Perdasdegogu y el parque eólico.

Elaboración propia a través de herramienta SIG.

El punto de inflexión de produjo en abril del 2016 cuando fue publicada la propuesta de una ampliación del parque, comprendiendo 9 nuevos aerogeneradores, 5 en el interior del territorio de Perdasdefogu y 4 en el de Ulassai. La propuesta encontró pleno consenso ya sea por parte de la administración que por parte de los ciudadanos. Durante el encuentro con la población, organizado siguiendo el proceso marcado por VIA, se asistió a una consenso por parte de una platea constituida mayormente por jóvenes, tirados por las oportunidades de empleo que estaban ofreciendo. Está claro como los dos contextos proporcionen un claro ejemplo de generación de un conflicto entre paisaje y desarrollo, en el cual el riesgo de manipulación es elevado.

La investigación mira por ella a la búsqueda de las diferentes actitudes de la población en relación con el tema de "paisaje y desarrollo" y a identificar posibles interrelaciones entre el comportamiento visto en relación a la propuesta del proyecto y la percepción del impacto y de sus beneficios.

El objetivo es el de identificar, sobre la base de los diferentes procesos activados a partir del parque eólico, las características de las poblaciones de Ulassai y Perdasdefogu, en referencia al conocimiento sobre las fuentes renovables, la posición y la disponibilidad para acrecentar la propia capacidad de evaluación. Se busca, de esta manera, una dependencia entre la afiliación a uno, en lugar de al otro contexto y la actitud adoptada hacia los beneficios y los impactos. 


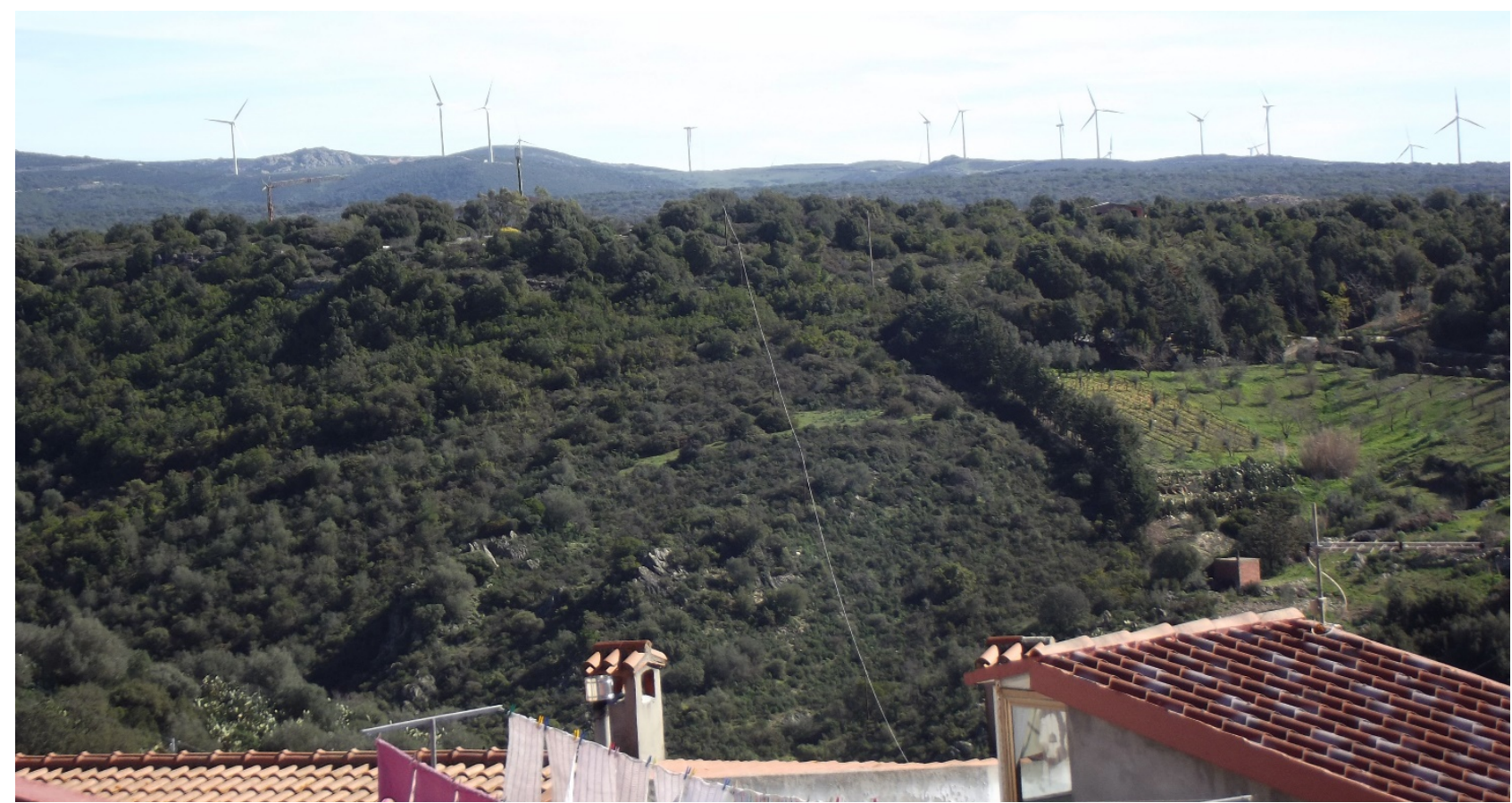

Gráfico 2: Vistas del parque eólico de Perdasdefogu.

Fuente propia: 08.03.2017

\subsection{El eje del conocimiento}

El análisis ha puesto en relación la auto-evaluación del conocimiento personal con los resultados del test verdadero/falso. Con el objetivo de distinguir a los individuos sobre la base de la percepción y del real conocimiento, han sido identificados cuatro casos principales

-"sabios" quien sabe de saber, y efectivamente conoce;

-"presuntuosos" quien afirma de saber más de lo que realmente sabe;

-"conscientes" quien sabe de no saber;

-"inseguros" quien minusvalora el conocimiento que en el realidad posee;

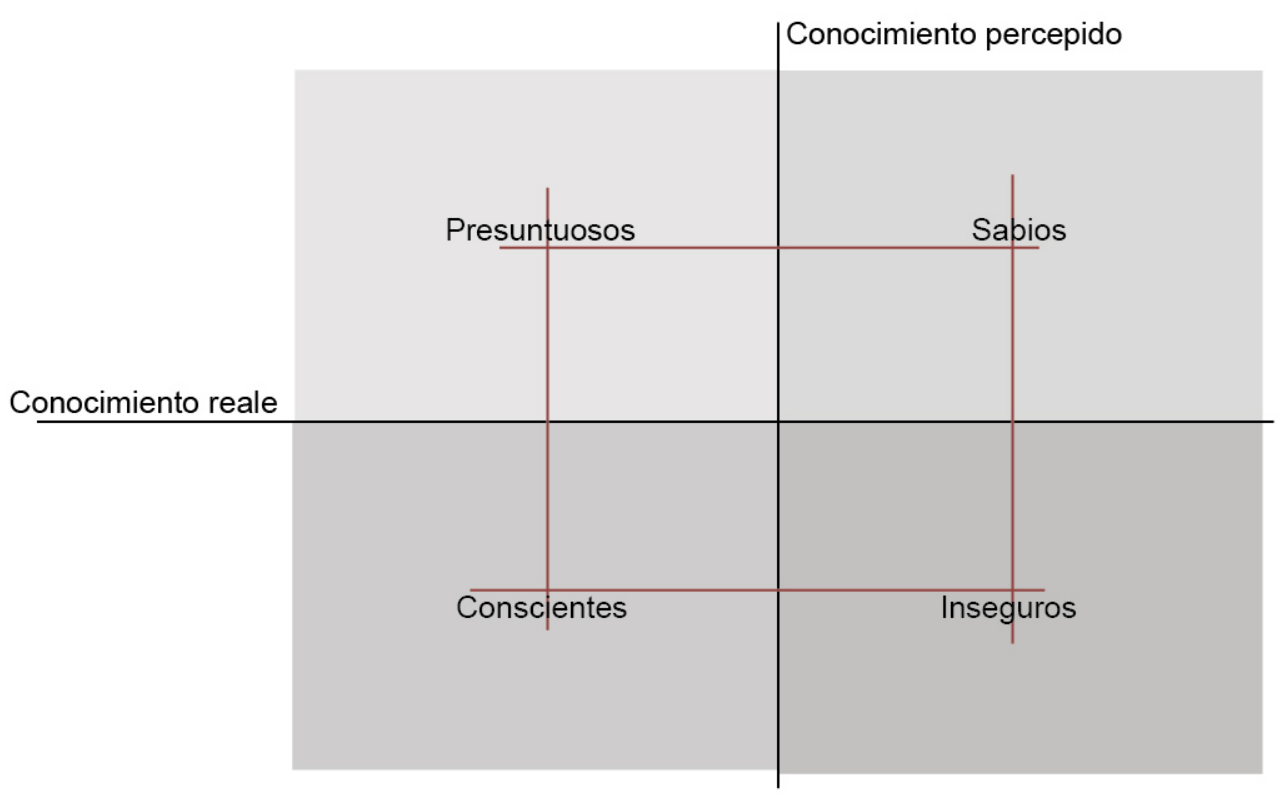

Gráfico 3: Esquema de asignaciones de las diferentes clases de conocimiento 
Poniendo en relación estas clases con la variable del pueblo de residencia, descubrimos que los dos están formados de poblaciónes similares por el nivel de conocimiento, real y percepido.

\subsection{El eje de la opinión}

La investigación relativa a la actitud adoptada por los entrevistados durante la evaluación de un proyecto de instalación FER resulta ser una parte fundamental del trabajo porque permite, indirectamente, de identificar los elementos sobre los cuales se basa la percepción de una intervención específica. Los resultados se las evaluaciones han sido puestas en relación la variable del Ayuntamiento de residencia, sacando a la luz una relevantes diferencia de comportamiento. De la comparación de los gráficos se puede ver como la comunidad ulassese, representada por la muestra obtenida, se encuentra dividida en dos partes respecto al proyecto menos impactante pero con beneficios nulos (Proyecto A), tendiendo hacia juicios más extremos (Pésimo, Escaso, Óptimo y Bueno) que hacia aquellos moderados. En Perdasdefogu, en cambio, los entrevistados tienes a evaluar el proyecto como Óptimo y Bueno, con un porcentaje que llega casi al $50 \%$. Con el proyecto intermedio la tendencia de estos últimos es todavía más clara al definir el proyecto como "óptimo", con un porcentaje del $32,30 \%$, contra poco más del $11 \%$ de votos "pésimo" y "escaso". Por el contrario, en Ulassai, la tendencia se invierte y los habitantes tienden a asignar mayormente un voto medio y bueno, con respectivamente el $24,51 \%$ y $37,25 \%$. El proyecto $C$ presenta el impacto más alto pero un consecuente y relevante aumento de los beneficios, hecho que lleva a los habitantes de Perdasdefogu a elegir, con un porcentaje de más del $50 \%$ de votos "óptimo" y un poco por debajo del $30 \%$ de "bueno". En Ulassai se observa una actitud parecida a aquella adoptada con el primer proyecto: la población está dividida en dos posiciones extremas, el $23,53 \%$ vota "pésimo" y el 29,41\% "óptimo". El resto de la población mantienes juicios intermedios distribuyéndose de manera bastante uniforme.

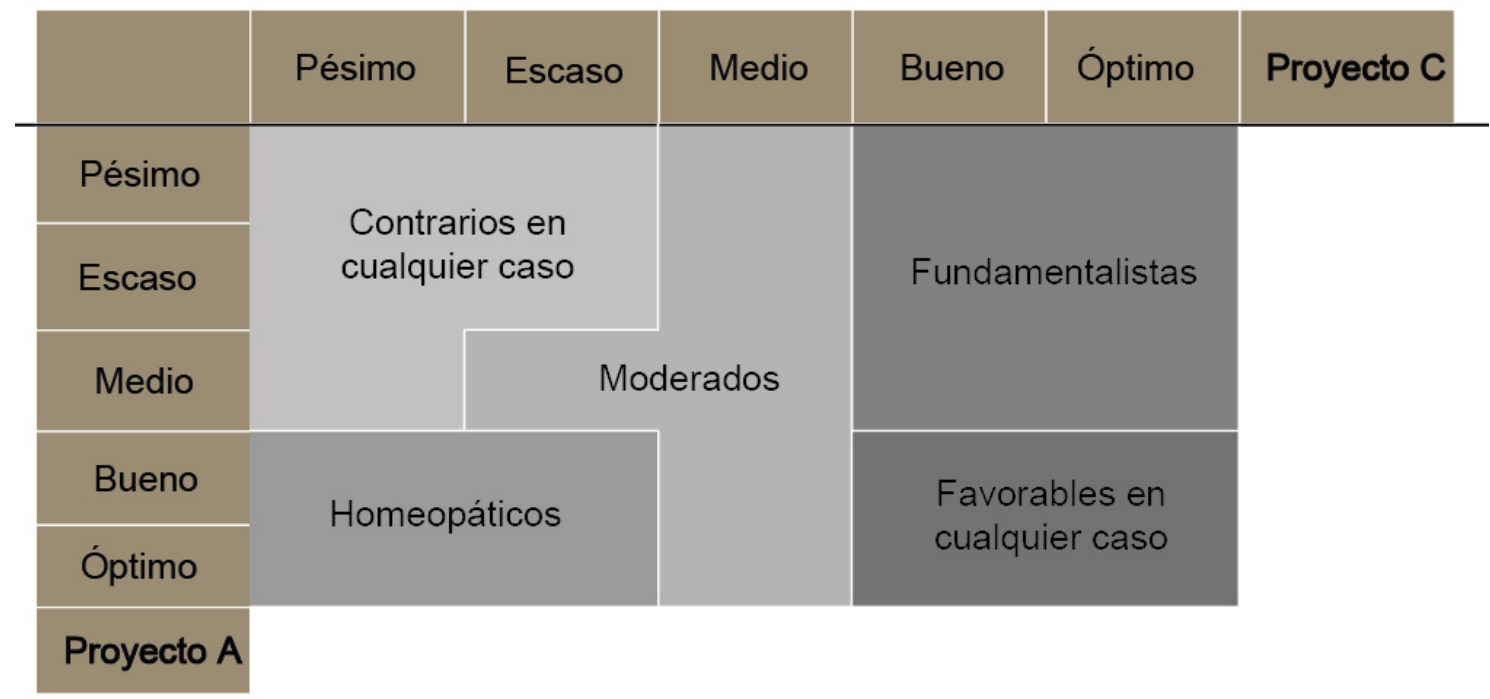

Gráfico 4: Esquema de asignaciones de las diferentes clases de opinión

Se decidió profundizar en la investigación partiendo de la observación de las respuestas de cada individuo respecto a los dos proyectos extremos, de manera que se pudiera comprender la actitud de cada uno e identificar una posible tendencia. Ha sido posible identificar cinco clases de opinión (Gráfico 4):

-"Contrarios en cualquier caso": los cuales evalúan ambos proyectos con votaciones negativas, mostrando su aversión hacia este tipo de proyectos, más allá de los posibles beneficios económico;

-"Favorables en cualquier caso": los cuales evalúan de manera positiva ambos proyectos, demostrando la propia pretensión a aceptar proyectos de instalaciones de cualquier dimensión sin importar el impacto generado;

-"Fundamentalistas": son aquellos que confieren una votación elevada al proyecto más agresivo y un voto 
bajo a aquel paisajísticamente menos impactante, revelando una actitud propensa a preferir una acción radical más que minimalista;

-"Homeopáticos": son aquellos que, opuestos a los fundamentalistas, aceptando impactos minimos y evalúan de manera fuertemente negativa el proyecto $C$.

-"Moderados": son aquellos que no tienden hacia posiciones extremas sino que se mantienen en un punto intermedio.

Se ha procedido analizando los datos obtenidos sobre la variable del Ayuntamiento y, somo se puede ver en el gráfico 5 , la diferencia de opiniones entre los dos contextos es particularmente relevante. En Perdasdefogu más del $43 \%$ de los entrevistados pertenecen a la categoría de Favorables, contra un $21,57 \%$ de Ulassai. Por la clase de Contrarios, en cambio, sólo el 3,75\% de los individuos de Perdasdefogu pertenecen a esta categoría y de Ulassai más del 19\%; el mismo fenómeno se observa en la categoría de los Homeopáticos. Otro porcentaje relevante de los habitantes de Perdasdefogu pertenece a la categoría de los Integralistas, con un porcentaje de casi el $37 \%$, mientras que los ulasseses se paran el $24 \%$. Al final, el $12 \%$ de los residentes de Perdasdefogu son moderados, contra el $19 \%$ de los ulasseses. De ve claramente como la opinión de la población de Ulassai está dividida en varias corrientes de pensamiento, mientras que en Perdasdefogu está mayormente difundida la corriente Favorable e Integralista.

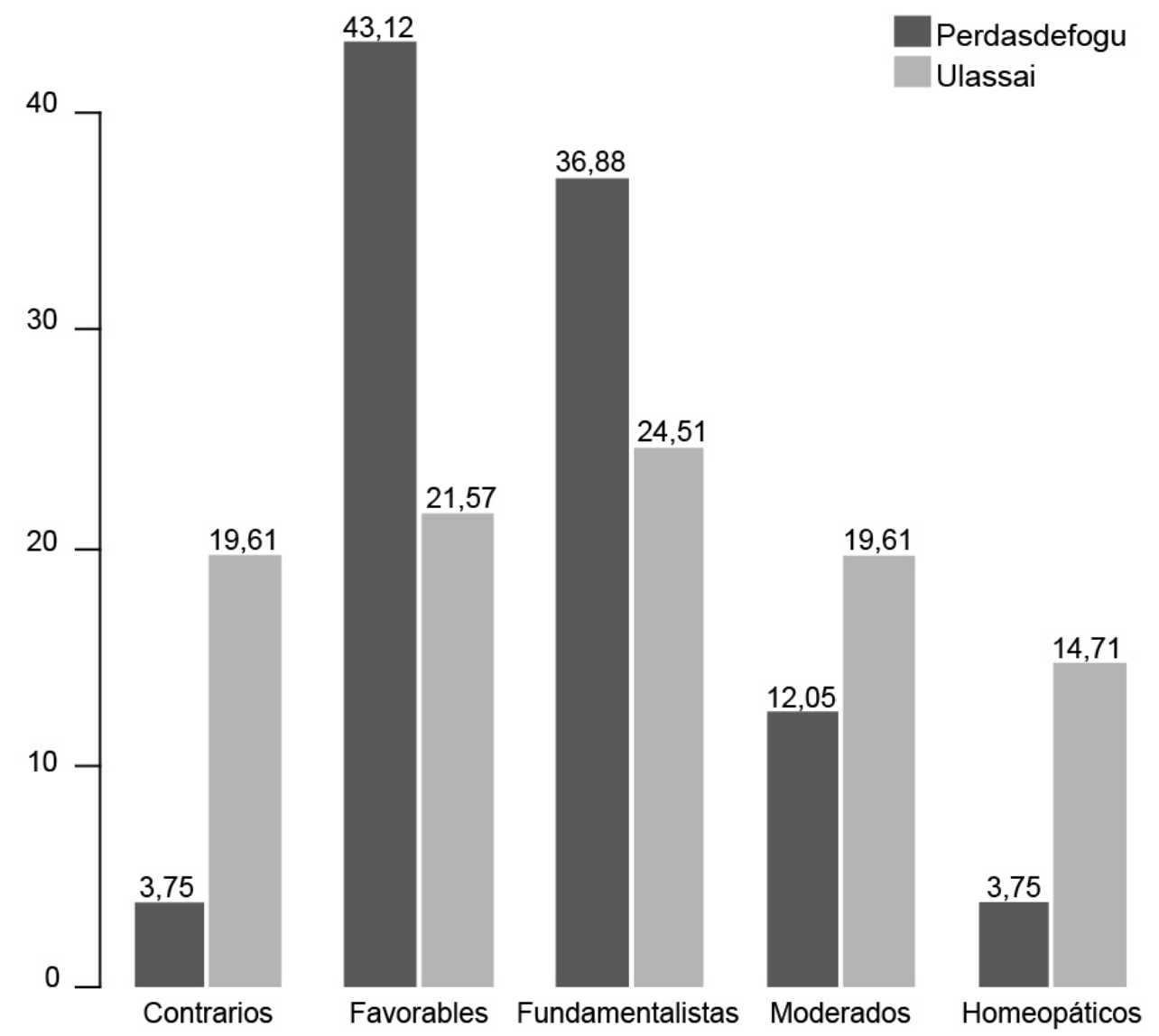

Gráfico 5: Histograma de la disposición de los individuos dentro de las diferentes opiniones en base a la variable del pueblo (\%)

\subsection{La percepción de los beneficios y de los impactos}

Con el objetivo de estudiar las opiniones de la población sobre la base de las instalaciones y de los beneficios percibidos, se ha analizado la percepción personal de los individuos a aquella que, según ellos, pertenece a la propia comunidad. En este caso la diferencia entre los dos pueblos es evidente respecto a la percepción del impacto sobre el paisaje, de los beneficios económicos y del impacto de empleo. El gráfico 6 muestra las 
respuestas de los entrevistados sobre la percepción personal sobre los efectos del parque sobre el paisaje, diferenciando entre los dos contextos analizados. No obstante en ambos pueblos los porcentajes mayores de entrevistados declaran no haber sido influencia de algún modo desde este punto de vista, en Perdasdefogu el $25,77 \%$ define los efectos sobre el paisaje negativo, contra un $11,66 \%$ que lo percibe como positivo. En Ulassai la tendencia es opuesta, con más de un $29 \%$ de los entrevistados que afirma que la propia vida ha sido influenciada positivamente de los efectos de paisaje del parque eólico, contra un $7,77 \%$ que ha sido influenciado negativamente.

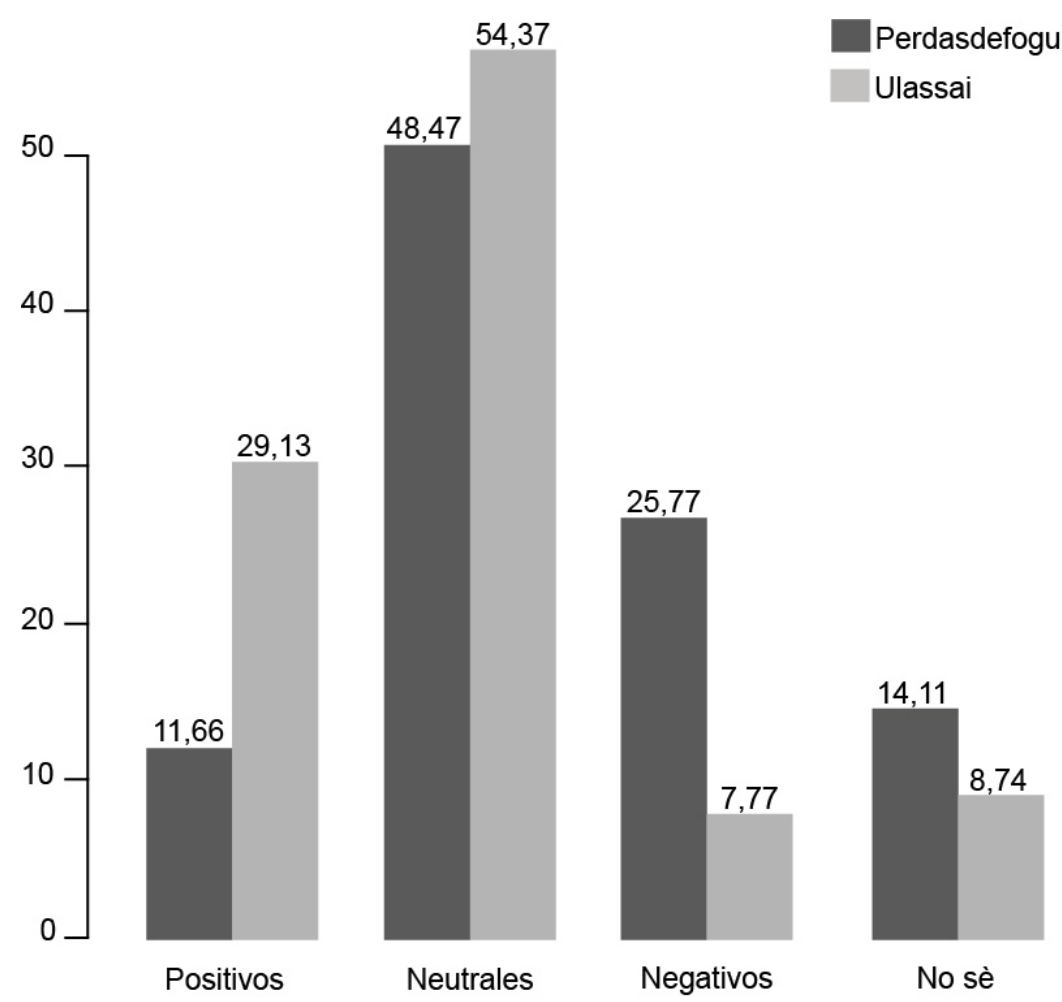

Grafico 6: Histograma de la percepción personal de los efectos sobre el paisaje, distinta para los dos pueblos (\%)

El tema de los beneficios económicos, como el previsto, saca a la luz relevantes disparidades entre los dos pueblos. El $87,50 \%$ de las personas de Ulassai afirman que la propia colectividad ha recibido influencias positivas del parque eólico desde el punto de vista de beneficios económicos, mientras que en Perdasdefogu el porcentaje se para en un $19 \%$. Más de la mitad de los entrevistados de Perdasdefogu declara que el propio pueblo no ha recibido ningún beneficio económico, contra menos del $2 \%$ de Ulassai (gráfico 7 ). 


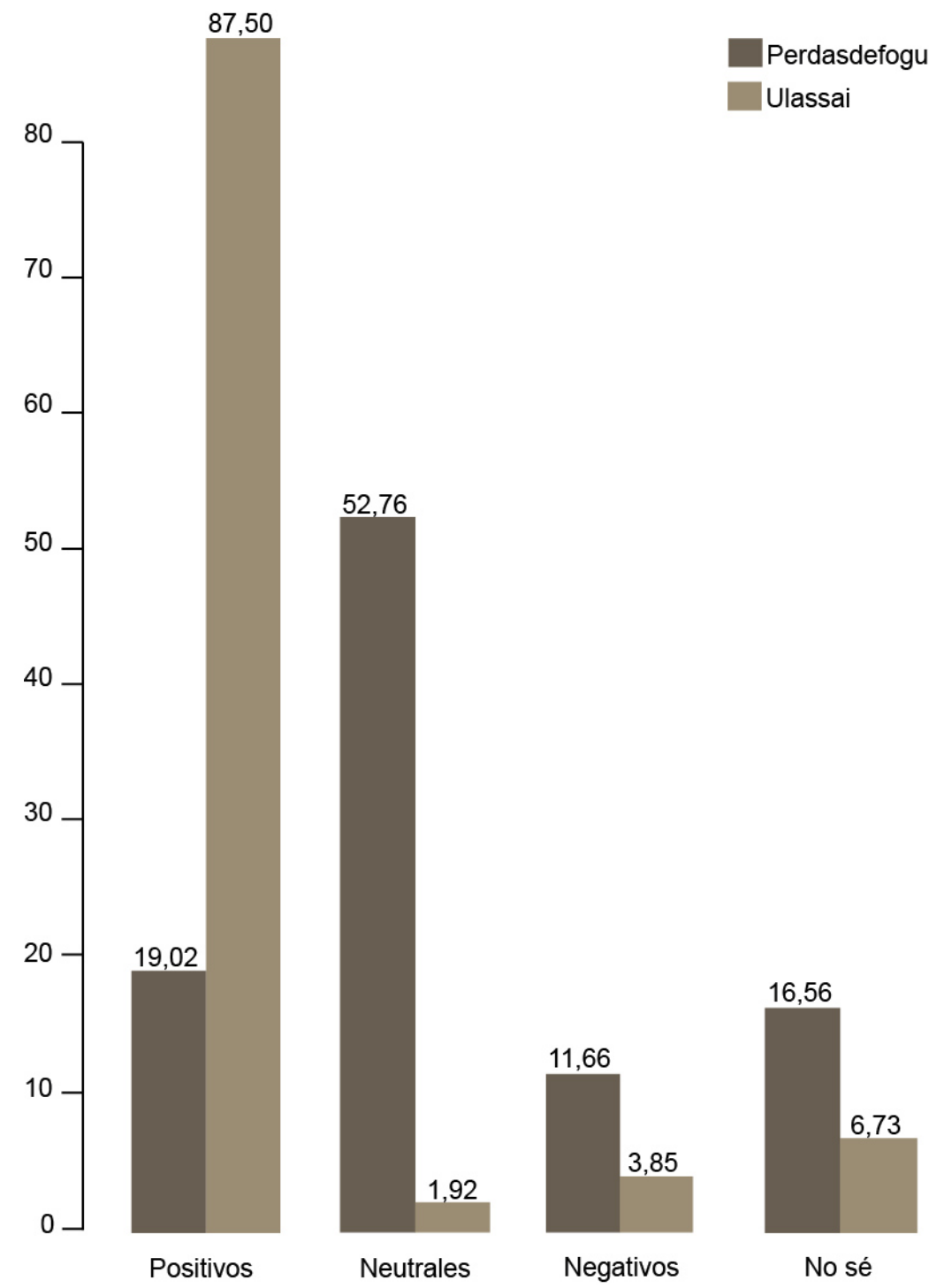

Gráfico 7: Histograma de la percepción de los efectos sobre la economía de la comunidad, distinta para los dos pueblos (\%)

La misma tendencia se observa en relación con el impacto de empleo, las cuales aparecen indiferentes por el $61,35 \%$ por la muestra perteneciente a Perdasdefogu, contra caso el $38 \%$ de Ulassai.

\subsection{La disponibilidad a la real implicación}

Para la última parte del análisis el cuestionario se ha concentrado en la disponibilidad de los entrevistados a entrar a formar parte de un proceso de implicación, investigando sobre cuánto piensan que pueda ser útil para mejorar su capacidad de evaluar proyectos de instalaciones FER. Ha sido investigada la percepción de la importancia que se atribuye a la población antes de la construcción del parque, la idea personal sobre la importancia que la participación de las personas puede o debería asumir en el proceso de evaluación, la utilidad de una campaña de información precedente a la presentación al público y la necesidad de un conocimiento específico para mejorar la capacidad de evaluación de estos proyectos. De los resultados se ha identificado una total apertura a la real implicación, con una tendencia parecida en ambos pueblos.

\subsection{Consideraciones finales}

Del análisis de los datos ha sido posible delinear específicas diferencias que existen entre los dos contextos, los cuales resultan en algún caso claramente dependientes del binomio Paisaje-Desarrollo, así como 
reconocido en algún Ayuntamiento, en referencia a los beneficios y al impacto percibido. El pueblo de Perdasdefogu asume una actitud separado en relación al componente paisajístico, tendiendo al desarrollo de las energías renovables, ignorando su impacto. Al contrario, en Ulassai, los habitantes demuestran tener posiciones heterogéneas, revelando un mayor "apego al paisaje".

Los resultados obtenidos permiten delinear un modelo basado sobre tres variables: Conocimiento, Opinión y Disponibilidad a la implicación, definiendo así una estructura con tres ejes (Gráfico 8). Cada individuo puede ser clasificado sobre la base de estas tres variables, definiendo el dibujo de un preciso triángulo. La figura obtenida de la unión de los tres puntos extremos representa un individuo que posee un buen nivel de conocimiento y que es consciente de ello, una opinión moderada respecto a la fuentes renovables, que tiene en cuenta el impacto en el paisaje y los beneficios económicos y una actitud totalmente abierta a la implicación.

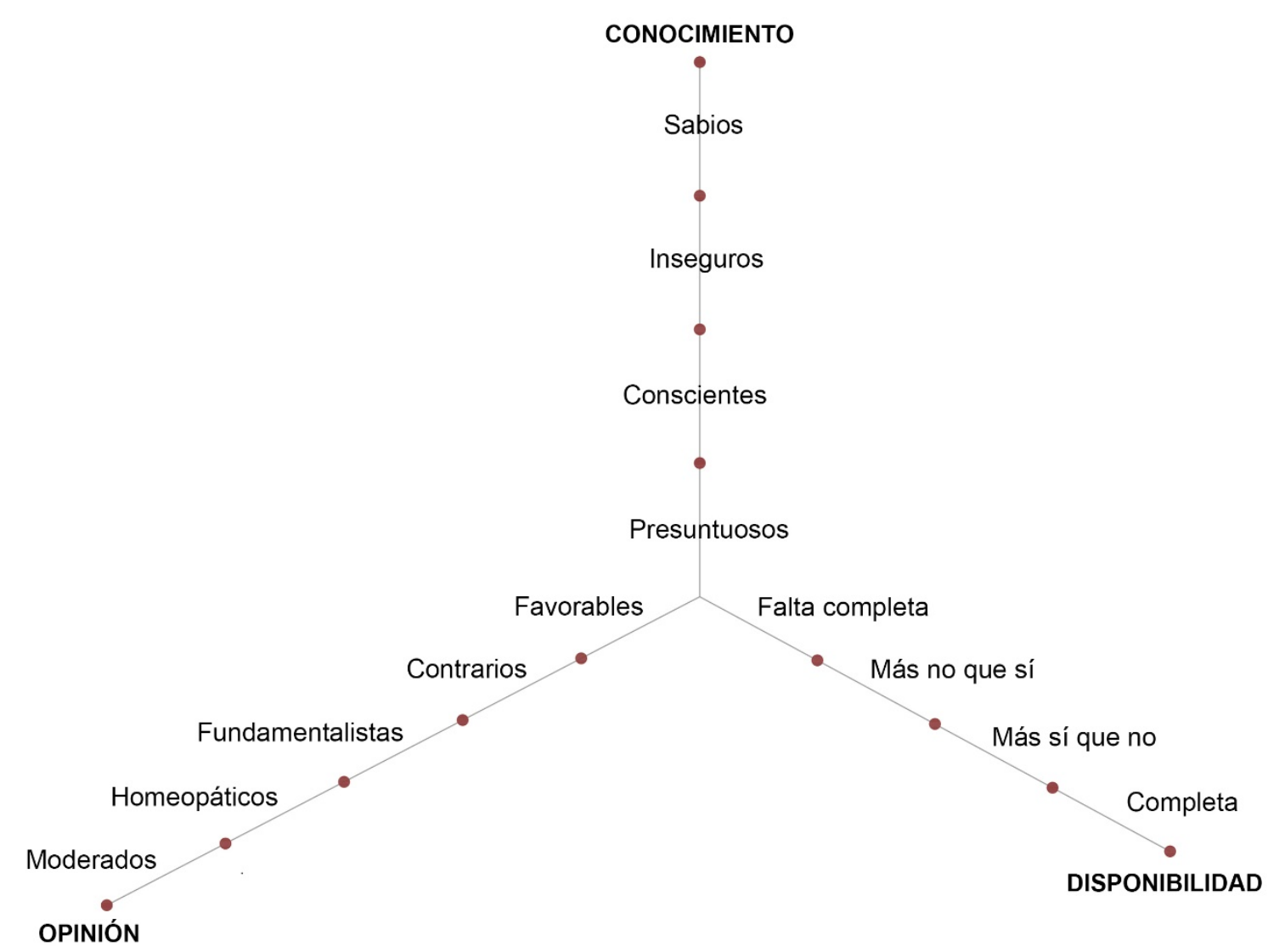

Gráfico 8: Estructura base del modelo

Para ambos casos los contextos han sido definidos con triángulos relativos a las dos categorías de individuos más numerosa. Una vez identificado el principal nivel de conocimiento, han sido determinadas las respectivas clases de opiniones, especificando las dos con el mayor porcentaje, definiendo pro ésto dos triángulos para cada clase conocimiento. Se ha decidido seguir con esta metodología distinguiendo en el concepto de "conocimiento" un mayor grado de manipulación legado a procesos sociales y actitudes personales. Aunque el conocimiento real redice la influencia de juicios personales.

Procediendo a la aplicación del modelo sobre el que los dos contextos analizados, se ha encontrado un límite relativo al eje de la "Disponibilidad a la implicación" que ha sido explorado con menos profundidad que los otros. Por ésto se ha tenido en cuenta el hecho que en cada caso analizado la población ha afirmado retener importante la propia participación en las fases de Evaluación Ambiental y el útil construir un conocimiento específico y por lo tanto crítico sobre el tema de las Energías Renovables. Por estos datos y los numerosos 
testimonios verbales, aunque sea necesaria una posterior profundización, se ha decidido mantener el punto sobre el eje de la Disponibilidad fijo sobre la categoría "completa disponibilidad".

Respecto al pueblo de Perdasdefogu se ha identificado un $32,51 \%$ de individuos pertenecientes a la clase de los "inseguros", a su vez constituidos por más del $80 \%$ de "favorables" e "fundamentalistas". Se delinea por ésto un primer gráfico que diferencia la componente de los "favorables en cualquier caso", que tiene un porcentaje más alto que los "fundamentalistas". A la categoría de "Conscientes" corresponde un 44,4\% de "fundamentalistas" y un 32,08\% de "favorables" (Gráfico 9).

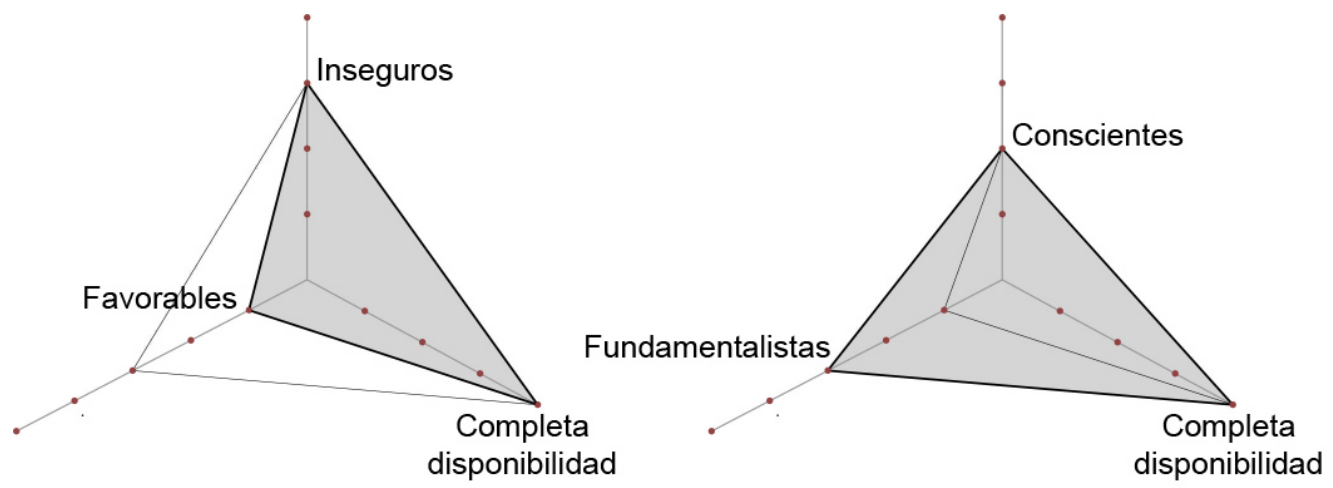

Gráfico 9: Modelo aplicado a la población de Perdasdefogu. El triángulo gris representa la clase de opinión más numerosa.

En Ulassai la clase de conocimiento de los "inseguros" contiene el $39,41 \%$ de las muestras, que resulta formada por el $32,50 \%$ de los "fundamentalistas" y por el $20 \%$ de "favorables". Los "sabios" son el $23,07 \%$ y están compuestos por un 33,3\% de "contrarios" y da un 25\% de "moderados" (Gráfico 10).
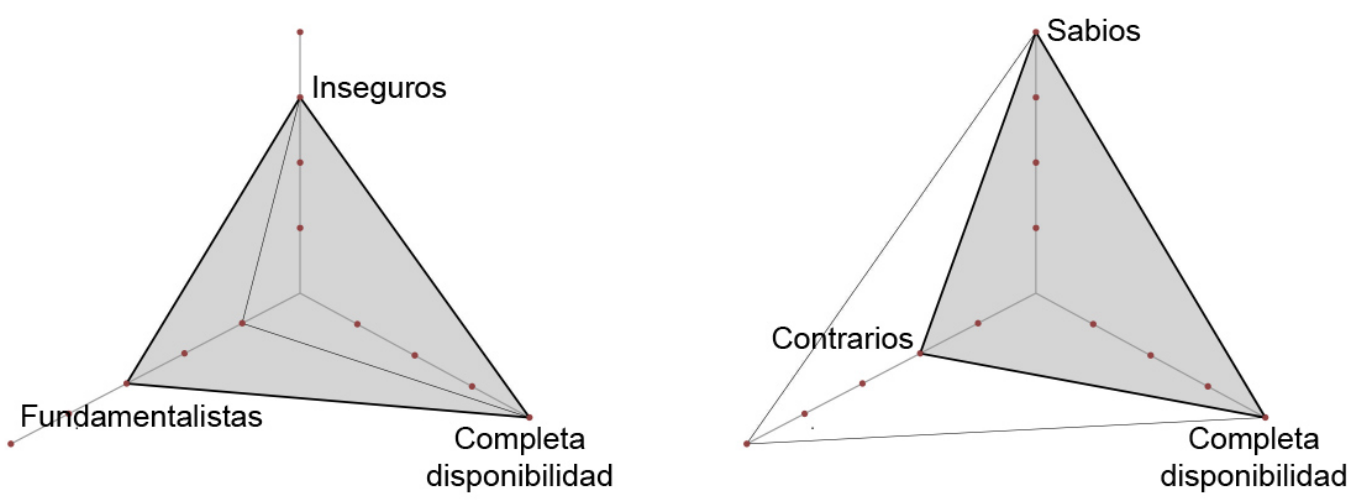

Gráfico 10: Modelo aplicado a la población de Ulassai

En relación a las figuras se puede constatar como la población de Ulassai sea mayormente heterogénea, sin asumir una posición específica, pero formando una serie de tipologías diferentes las unas de las otras. En Perdasdefogu la conformación de las tipologías de individuos es más o menos parecida, tendiendo en cada grado de conocimiento hacia una posición de apoyo al desarrollo más que a la salvaguardia del paisaje.

\subsection{Propuestas}

Del análisis del proceso de VIA se ha permitido la identificación de algunos límites en ella definición de la fase de consulta, los cuales son generados de un riesgo de manipulación de la información y por lo tanto de la percepción de las personas sobre los beneficios y el impacto de mi proyectos de instalación FER. Resulta necesaria una fase precedente a la presentación al público que esté compuesta por diferentes procesos de implicación de las personas y que permita construir en el interior de la comunidad un conocimiento de base sobre el tema, en referencia a los impactos y beneficios de las instalaciones no fósiles. El urbanista asume 
un rol fundamental para tratar junto con la población el tema de la tutela del ambiente global, garantizando el desarrollo local y valorizando el paisaje. La fuerte correlación de tal argumento con las peculiaridades de cada contexto llaman a la necesidad de una metodología de investigación y de acciones que sean adecuadas para cada una de las realidades. En este trabajo se ha querido proponer un modelo que, por su flexibilidad, resulta ser adaptable a cada territorio en el cual el paisaje, el ambiente y el desarrollo de las energías renovables son llamadas en causa y puestas en relación.

El objetivo del modelo es aquel de identificar las principales tipologías de individuos para poder construir estrategias específicas de implicación.

El dibujo de cada tipología de individuos se basa sobre los ejes del conocimiento, de la opinión y de la disponibilidad a la implicación, y sobre la base de tales características de los sujetos, se puedan definir acciones de intervención. Respecto al eje del conocimiento, constituido por la relación entre conocimiento percibido y real, en el caso de una población mayormente compuesta por individuos "sabios", no será necesario adoptar una estrategia de mejoramiento del conocimiento, concentrando las acciones sobre el eje de la opinión. En el caso de una población constituida por "inseguros" o "presuntuosos", los cuales no tienen ideas claras del propio nivel de conocimiento, será preferible activar una campaña de información indirecta que cuente el estado real de las cosas. La categoría "conscientes", Ilama a una acción más directa y preparada para construir un conocimiento que, en la mayor parte de los casos, no se posee por falta de interés. La modalidad de difusión de la información debe adaptarse a la composición de la población, en referencia a la edad, al grado de interés por las políticas públicas, al nivel de conocimiento y experiencia sobre el tema de las fuentes renovables.

Al eje de la opinión se conectan las acciones y estrategias que se ponen como objetivo llevar la actitud de las personas hacia un intento de integración entre las cuestiones de la tutela del paisaje, de la protección del ambiente y de la garantía de un desarrollo de la comunidad. Pro este motivo las posiciones más radicales como "favorable en cualquier caso" o "contrario en cualquier caso" van orientadas hacia una visión más moderada, que tenga en cuenta todos los elementos del territorio y de la relación entre ellos. En el caso de una supremacía de la categoría de "favorable" o "fundamentalistas", notablemente orientados hacia un desarrollo de la tecnología dejando a un lado el impacto sobre el paisaje, se ha previsto una serie de acciones de construcción de una sensibilización para la tutela y valorización del paisaje. El contexto de Perdasdefogu es un claro ejemplo de una comunidad que ha perdido casi totalmente el vínculo con el paisaje, llegando a atribuirle principalmente el significado de elemento de explotación para generar beneficios económicos. La conexión con el territorio se reconstruye acompañando a la población al redescubrimiento de la componente del paisaje, con eventos y actividades que miran hacia la reapropiación del territorio y a la identificación de los significados de los lugares que han sido olvidados. Se ententa de esta manera de construir en la población un vínculo con su propio territorio que haga a las personas reales protagonistas de la valorización del paisaje. Al contrario, si tuviera que verificarse una supremacía de la categoría de "contrarios" o "homeopáticos" que tienden claramente hacia una defensa del paisaje más que hacia las tecnologías renovables, la estrategia sería aquella de limar la actitud cerrada hacia las nuevas posibilidades de desarrollo. Se refiere a acciones desde un punto de vista global, se busca así desencadenar un sentimiento de responsabilidad.

El eje de la disponibilidad a la implicación es necesario para dar una idea de las modalidades con las que será necesario actuar sobre los otros dos ejes. Si se registra una baja voluntad de implicación, se deberá construir una serie de estrategias a largo plazo y menos directas, basadas sobre actividades y eventos que introduzcan mano a mano al tema del desarrollo de las energías renovables.

Acciones integradas de construcción de un conocimiento crítico, de una aproximación integral entre los términos de paisaje y de energía y una actitud abierta a la real implicación en los procesos de evaluación, reducirán el riesgo de manipulación durante la fase de presentación al público. Resulta por esto fundamental la inserción de este proceso durante la fase precedente a la fase de Evaluación del Impacto Ambiental, de manera que la población madure un conocimiento crítico y se ponga de frente a un proyecto de instalación FER con una mejor capacidad de evaluación. 


\section{CONCLUSIONES}

En un panorama de desarrollo de las tecnologías de producción de energía de fuentes renovables, la relación con el paisaje resulta ser un tema fundamental de la planificación sostenible del territorio. La investigación sobre los conflictos entre Paisaje, Ambiente y Desarrollo, generados de la inserción de estos nuevos elementos, no puede prescindir de un análisis relativo a la repercusión en la sociedad. Con el fin de que la propia transición energética sea compartida y no denegada es necesario hacer que las personas asuman un rol activo en la evaluación de los impactos y de los beneficios.

Han sido encontrados los límites de los instrumentos de Evaluación de los proyectos relativos a las instalaciones FER, legados a la posibilidad de manipulación del comportamiento de las posiciones asumidas por los individuos. Por esto se ha querido hablar, más que de participación, de real implicación: la población se convierte en protagonista en la valorización y salvaguardia de su territorio. Para añadir a este objetivo elementos esenciales resultan ser un conocimiento específico sobre el tema de las energías renovables y una sensibilidad respecto a la tutela del paisaje, de manera que las personas evalúan estos proyectos con una aproximación integral entre Paisaje, Ambiente y Desarrollo.

Este trabajo intenta identifica un instrumento de análisis de la población involucrada en los conflictos generados por las Energías Renovables, identificando varias tipologías de individuos, sobre la base de cuales se ha definido una estrategia adecuada de implicación. La investigación sobre el territorio de Ulassai y Perdasdefogu ha permitido realizar una aplicación del modelo, sacando así a la luz las diferentes actitudes de la población. La metodología propuesta, basándose sobre las peculiaridad del contexto, resulta ser flexible y, por lo tanto, repetible en cualquier territorio interesado en este tipo de conflicto.

\section{BIBLIOGRAFIA}

AA. VV. (1989). I/ futuro di tutti noi. Milano: Bompiani

BOURASSA, S.C. (1990). A paradigm for landscape aesthetics. Environment and Behaviour (Connecticut), 22(6), 787-812

Convención europea del Paisaje (2000), Consejo de Europa. Firenze. Octubre 2000

Costituzione della Repubblica italiana (1948), Assemblea Costituente. Diciembre 1947

CUBASCH, U. et al. (2001). Projections of future climate change. En J. T. HOUGHTON et al., Climate Change 2001: The Scientific Basis (527-582). New York: Cambridge University Press

Decreto Legislativo n. 152, "Norme in materia ambientale", publicado en la Gazzetta Ufficiale n. 88 del 14 aprile 2006 - Supplemento Ordinario n. 96

DI FIDIO, R. (1996). Dizionario di ecologia. Milano: Pirola Direttiva 2009/28/CE, Parlamento europeo e del Consiglio. Abril 2009

D. Lgs. n. 42/2004, Codice dei beni culturali e del paesaggio, ai sensi dell'articolo 10 della legge 6 luglio 2002, n. 137. Publicado en la Gazzetta Ufficiale n. 45, febrero 2004

FERRARO, L. (2012). Costituzione, tutela del paesaggio. Forum di quaderni costituzionali (web), 10 junio 2012

KAPLAN, S. (1979). Perception and Landscape: Conception and Misconception. En AAVV, Proceeding of Our National Landscape (241-248). USDA Forest Service

$\mathrm{KOH}$, J. (2013). On a landscape approach to design: and ecopoetic interpretation of landscape. Wageningen University Press 
IPCC (2013). Climate Change 2013: The Physical Science Basis. Contribution of Working Group I to the Fifth Assessment Report of the Intergovernmental Panel on Climate Change [Stocker, T.F., D. Qin, G.-K. Plattner, M. Tignor, S.K. Allen, J. Boschung, A. Nauels, Y. Xia, V. Bex and P.M. Midgley (eds.)]. Cambridge, New York, Melbourne, Madrid, Cape Town, Singapore, São Paolo, Delhi, Mexico City: Cambridge University Press

MAGONI, M. (2013). Energia e paesaggio al tempo dei cambiamenti climatici. REAL CORP 2013 (Roma), 2023 majo, 1169-1176

MARCHIGIANI E. y PRESTAMBURGO, S. (2010). Energie rinnovabili e paesaggi. Strategie e progetti per la valorizzazione delle risorse territoriali. Milano: Franco Angeli

MÖLLER, B. (2003). Least-cost allocation strategies for wood fuel supply for distributed generation in Denmark - a geographical study. International Journal of Sustainable Energy, 23(4): 187-197

MURA, M. y STRAZZERA, E. (2013). Vento, sole, paesaggio: beni comuni rinnovabili. Cosa pensano $i$ cittadini delle energie rinnovabili. Cagliari: CUEC Editrice

PASQUALETTI, M. J. (2012). Reading the Changing Energy Landscape. En S. STREMKE y A. DOBBELSTEEN, Sustainable energy landscapes: designing, planning and development (11-44). Boca Raton: CRC Press

PETERSON, R. (2006). Why Mami Wata Matter: Local Considerations for Sustainable Waterpower Development Policy in Central Africa. Local Environment 11, 1 (Enero): 109-125

RIFKIN, J. (2011). La terza rivoluzione industriale. Milano: Mondadori

SALZANO, E. (1998). Fondamenti di urbanistica. Bari - Roma: Editori Laterza

SERENI, E. (1984). Storia del paesaggio agrario italiano. Roma-Bari: Editori Laterza

SIJMONS, D. y VAN DORST, M. (2012). Strong Feelings: Emotional Landscape of Wind Turbines. En S. STREMKE y A. DOBBELSTEEN, Sustainable energy landscapes: designing, planning and development (4567). Boca Raton: CRC Press

SMITS, M. (2002). Monster Treatment: The Cultural Domestication of New Technology. Amsterdam: Boom Publishers

STREMKE, S. y KOH, J. (2011). Integration of Ecological and Thermodynamic Concepts in the Design of Sustainable Energy Landscapes. Landscape Journal, 30(2): 194-2013

SUKKUMNOED, D. (2003). Distributed generation and centralized power system in Thailand: Conflicts and solutions. International Journal of Sustainable Energy, 4 (12): 199-206

VAN TIL, J. (1979). Spatial Form and Structure in a Possible Future: Some Implications of Energy Shortfall for Urban Planning. Journal of American Planning Association, 45 (3): 318-329

YÜKSEL, I. (2007). Development of hydropower: A case study in developing countries. Energy Sources Part B: Economics, Planning \& Policy, 2: 113-121

Fuentes electrónicas

https://ec.europa.eu/clima/policies/strategies/2020_it\#tab-0-0 (Consulta: 21/12/2016)

http://www.roadmap2050.eu/ (Consulta: 22/12/2016) 
https://www.c2es.org/international/negotiations/cop21-paris/summary (Consulta: 22/12/2016)

http://ec.europa.eu/eurostat/en/web/products-press-releases/-/8-10032015-AP (Consulta: 28/12/2016)

https://www.legambiente.it/contenuti/comunicati/comuni-rinnovabili-2016-I-innovazione-nei-territori-un-futuroenergetico-rinnov (Consulta: 05/01/2017)

http://demo.istat.it/bilmens2015gen/index.html (Consulta: 26/01/2017)

http://comune.ulassai.og.it/index.asp (Consulta: 01/02/2017)

http://comune.perdasdefogu.nu.it/hh/index.php (Consulta: 01/02/2017) 